Hossein M. Shodja*, Maryam Tabatabaei, Ladan Pahlevani and Alireza Ostadhossein

\title{
Diffusion of a self-interstitial atom in an ultrathin fcc film bonded to a rigid substrate
}

\begin{abstract}
The determination of the interstitial sites and saddle points corresponding to the diffusion of an interstitial atom in ultrathin face-centered cubic (fcc) film is of particular interest. The outcome is strongly influenced not only by the orientation of the free surface but also by the location of the defect with respect to the free surface and film-rigid substrate interface. In this article, an atomicscale simulation is conducted to analyze the effects of depth on the out-of-plane interstitial mechanism of diffusion. To ensure reasonable accuracy and numerical convergence, the atomic interaction up to the second-nearest neighbor is considered. The $a b$ initio examination of the above-mentioned problem associated with thin films requires a large supercell and is computationally time consuming. However, for the sake of demonstration, the values of the barrier height energy pertinent to a diffusing self-interstitial atom in the bulk material are computed using both the first principles density functional theory (DFT) and the developed technique, indicating reasonable correspondence.
\end{abstract}

Keywords: barrier height energy; first principles density functional theory (DFT); interstitial mechanism of diffusion; lattice statics method.

\footnotetext{
*Corresponding author: Hossein M. Shodja, Department of Civil Engineering, Sharif University of Technology, PO Box 11365-9313, Tehran, Iran, e-mail: shodja@sharif.edu

Hossein M. Shodja and Ladan Pahlevani: Institute for Nanoscience and Nanotechnology, Sharif University of Technology, PO Box 111559161, Tehran, Iran

Maryam Tabatabaei: Department of Civil Engineering, Sharif University of Technology, PO Box 11365-9313, Tehran, Iran Alireza Ostadhossein: Department of Engineering Science and Mechanics, The Pennsylvania State University, W 305 Millenium Science Complex, University Park, PA 16802, USA
}

\section{Introduction}

The diffusion phenomenon, due to its great influence on the physical and mechanical properties of solids, has been of great interest. Understanding the alterations of properties such as oxidation, creep, annealing, and such requires knowledge of the diffusion processes through the crystal lattice of interest. Moreover, diffusion is closely related to the way atoms move through solids, and knowledge of the process thus helps in the comprehension of the movements of point defects $[1,2]$. Some frequently observed mechanisms of diffusion in crystalline solids are interstitial, interstitialcy, and vacancy mechanisms. The first refers to the situation where an interstitial atom jumps to the nearest neighbor interstitial site. However, in the second, the interstitial atom substitutes the neighbor host atom by pushing it to the nearest interstitial site. In the third mechanism, the atom adjacent to a vacancy jumps into the vacant site and consequently leaves a vacant site behind. The barrier height energy is one of the important parameters in the study of diffusion of point defects. This parameter is defined as the energy required to surmount the barrier to the atomic movement in a diffusion mechanism. Nowadays, ultrathin films have an important role in achieving reliable microelectromechanical and nanoelectromechanical systems. Thus, it is very critical to study the effects of the free surface and the filmrigid substrate interface on the interstitial mechanism of diffusion as a relevant common problem. Surprisingly, the previous works addressing diffusion are largely pertinent to the case of bulk material.

Halicioglu and Barnett [3], using simulation, computed the formation and diffusion energies of intrinsic interstitials in Si substrates under strain condition. They incorporated Stillinger and Weber's empirical potential function, which is suitable for Si species, into their statics method. Xia et al. [4] studied the interstitial mechanism of diffusion for helium atoms in perfect bulk nickel and palladium via the molecular dynamics method. They described the interactions between metal atoms and between a helium atom and a metal atom using a modified analytic embedded-atom model and a Morse potential, respectively. They first calculated the diffusion coefficients using the jump-frequency method, and then the barrier height energy was obtained by fitting to the Arrhenius relation. Ortega et al. [5] considered selfdiffusion in bulk aluminum and nickel by simulating a crystallite consisting of $N$ atoms and $n$ vacant sites. The tracer self-diffusion Arrhenius relation was used in their calculations. The atomic interactions were represented using many-body potentials. In their work, the energies 
and vibrational entropies connected to the formation and migration of vacancies and divacancies were calculated by molecular static, and the vacancy diffusion mechanism over a wide range of temperature was studied using the molecular dynamic technique. Recently, Haftbaradaran et al. [6] proposed a continuum model of strongly coupled diffusion, stress, and solute concentration; they calculated the material properties by considering the pertinent atomistic model. For the verification of the continuum model, they used atomistic simulation of diffusion of $\mathrm{H}$ in single-crystal face-centered cubic (fcc) Ni. Their atomistic model is based on the embedded atom method potentials given by Angelo et al. [7]. The ab initio study of diffusion is not widespread due to high computational cost; simulations associated with thin films would be more expensive than those of bulks. LaBrosse et al. [8] calculated the vacancy formation energies and the diffusion barrier of an isolated vacancy in bulk $\mathrm{Co}, \mathrm{Fe}$, and Ni via the first principles. They examined the effect of surface intrinsic energy due to the presence of a vacancy. They also considered the substitutional tungsten diffusion in fcc bulk cobalt.

When the interstitial atom is the same as those in the host lattice, it is referred to as the self-interstitial defect. Although, under normal conditions, the presence of selfinterstitials in comparison with vacancies is relatively rare in metals, in high-energy radiation environments [9] and in ion implantation [10], their presence is both abundant and of great significance. For instance, in nuclear reactors, the collision of high-energy neutrons on metallic components results in the formation of large densities of self-interstitials. The diffusion of these point defects in thin film metals, due to its associated adverse effect on the service life of the film, is of major concern. The present work is devoted to the examination of the effects of the film free surface and the film-rigid substrate interface on the octahedral self-interstitial atom (OSIA) diffusion and the corresponding barrier height energy. Using lattice statics with an appropriate empirical potential function, the ultrathin fcc film on a rigid substrate can be effectively simulated with a reasonable number of atoms. Meanwhile, the simulation of a thin fcc film of about six-latticeparameter-thick using first principles density functional theory (DFT) requires the consideration of a rather large supercell (approx. 240 atoms), which is computationally very expensive. Fortunately, to model the fcc bulk in the presence of an interstitial atom, it is affordable to use the first principles DFT, using a supercell consisting of 65 atoms (see Section 3.1). The computed values of the barrier height energy for the interstitial mechanism of diffusion by the first principles DFT and the proposed lattice statics approach are in reasonable agreement, revealing that the developed method can be used to address the suggested problem.

\section{Energy considerations and computational details}

Consider an ultrathin film made of fcc crystal adhered to a rigid substrate. Set the origin of the Cartesian coordinates at the center of the specimen consisting of $N$ atoms. The periodic boundary condition is applied along the $x_{i}$ and $x_{2}$-directions parallel to the free surface, and the atoms on the bottom plane are kept fixed. The model involves an OSIA that occupies a site other than the host lattice sites (Figure 1). The concept for the calculation of the barrier height energy for the interstitial mechanism of diffusion is briefly discussed in Section 2.1. The methodology for calculating the total energy of the system is explained in Section 2.2.

\subsection{Calculation of the barrier height energy for the interstitial mechanism of diffusion}

The interstitial mechanism of diffusion for an fcc lattice with the cubic axes of the conventional unit cell occurs along the family of $\langle 110\rangle$. For further clarification, the interstitial sites within the (001) plane of a group of spheres packed into an fcc domain and within an fcc unit cell are shown in Figures 2A and B, respectively. Arrows

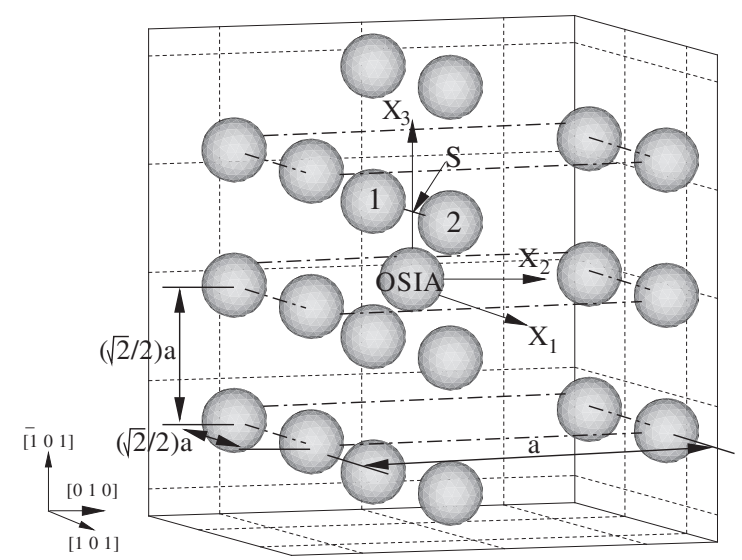

Figure 1 Nomenclature of an fcc domain containing an OSIA. Point $S$ is midway between the host atoms labeled 1 and 2, and $a$ is the lattice constant of the crystal. 
in Figure 2A indicate the possible paths of an OSIA within the family of $\{100\}$ planes to jump from one interstitial site to another. The octahedral coordination geometry is also illustrated in Figure 2B. In the present study, the crystallographic orientation of a thin fcc film is taken in such a way that the [101] direction is perpendicular to its free surface and the [101] and [010] directions are parallel to it. The Cartesian axes $x_{1}, x_{2}$, and $x_{3}$ coincide, respectively, with the [101], [010], and [101] directions, as shown in Figure 1. The dimensions of the specimen are $15 \sqrt{2} / 2 a, 11 a$, and $6 \sqrt{2} a$ along the $x_{1}^{-}, x_{2}-$, and $x_{3}$-axes, respectively, where $a$ is the lattice constant of the crystal. The film thickness is $6 \sqrt{2} a$ in the [101] direction, and the total number of atoms is approximately 4125 . With the above-mentioned conventions, $x_{3}=3 \sqrt{2} a$ indicates the top free surface of the film, and there is a perfect bonding between the bottom atomic plane $\left(x_{3}=-3 \sqrt{2} a\right)$ of the film and a rigid substrate.

To study the diffusion of an interstitial atom in a thin fcc film, after the relaxation of the defect-free film, an extra atom is inserted in one of the interstitial sites in the middle of the specimen. The interstitial mechanism of

A
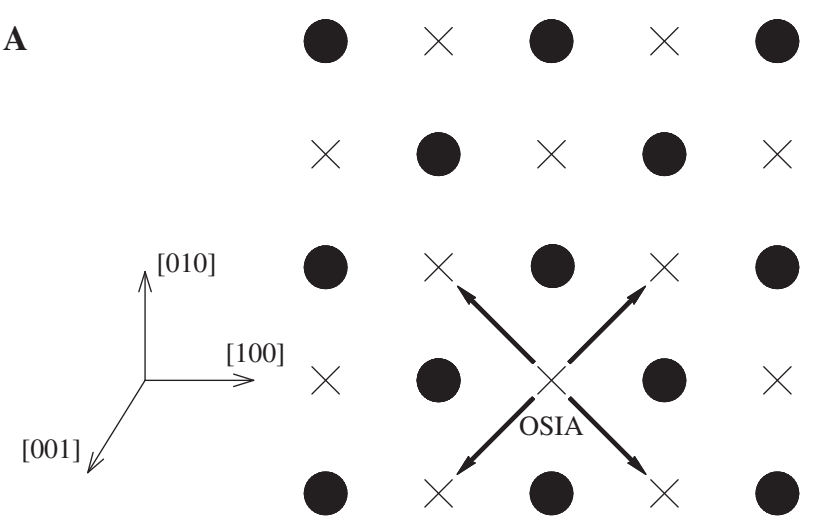

B

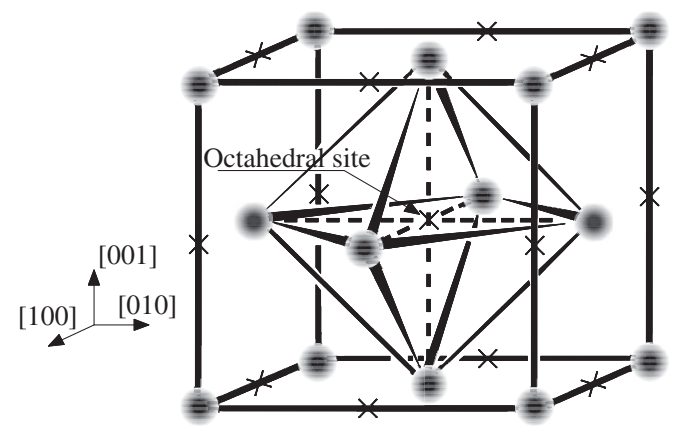

Figure 2 The $<110>$ family is clearly the migration path of the OSIA. The possible position of the interstitial site within the host fcc (A) (001) plane and (B) a unit cell demonstrating the octahedral site is indicated by $\times$. diffusion in fcc materials is such that an interstitial atom moves from its equilibrium site to the saddle point and subsequently to the nearest equilibrium site $[1,2]$. The saddle point is referred to a pick point connecting the two adjacent energy wells [11]. It should be mentioned that the diffusing atom is constrained in such a way that it can execute its normal vibration only in the plane perpendicular to the migration path while all degrees of freedom of all surrounding atoms are allowed to be fully relaxed [1, $2,12]$. The barrier height energy, $\Delta Q$, along the diffusion path is evaluated as [3]

$$
\Delta Q=E^{S}-E^{I},
$$

where

$$
E^{\alpha}=E_{T}(N+1, \alpha)-\frac{N+1}{N} E_{T}(N, 0), \quad \alpha \equiv I \text { or } S .
$$

In these equations, $E^{S}$ and $E^{I}$ denote the formation energies of the interstitial atom at the saddle point and at the interstitial site, respectively. $E_{T}(j, \alpha)$ and $E_{T}(j, 0)$ are the total energies of the specimen consisting of $j$ atoms; the former energy is related to the specimen with an interstitial atom at the interstitial site, $\alpha \equiv I$, or at the saddle point, $\alpha \equiv S$, whereas the latter energy is pertinent to the defectfree specimen. Among the out-of-plane paths, toward the free surface and the rigid substrate, the preferable diffusion path is the one for which $\Delta Q$ is a minimum. By considering an appropriate potential function, the effects of the free surface and the film-rigid substrate interface are intrinsically included in $E_{T}$

\subsection{Computational technique}

As it was alluded to, the interstitial atom moves along the diffusion path by jumping through interstitial sites step by step, allowing the surrounding atoms to continually readjust their positions at each step. Moreover, calculations are conducted to represent the low temperature limit. Therefore, the problem is essentially a static one, which can be examined using a suitable potential function in the framework of lattice statics. In this approach, the minimization of the total energy of a system consisting of $N$ atoms leads to

$$
\mathbf{K u}=\mathbf{f},
$$

where $\mathbf{K}$ is the stiffness matrix, $\mathbf{u}$ is the displacement vector, and $\mathbf{f}$ is the force vector, which accounts for the interaction of each atom with its first and second neighbors. $\mathbf{f}$ and $\mathbf{K}$ are obtained by consideration of the first 
and second derivatives of the total potential energy of the system with respect to the atomic position. The displacement vector is determined as $\mathbf{u}=\mathbf{r}-\mathbf{r}^{0}$, where $\mathbf{r}^{0}$ is the initial atomic configuration and $\mathbf{r}$ is the atomic equilibrium position vector after relaxation. The system is assumed to be relaxed when its total potential energy, $\Phi_{\mathrm{T}}$ is no longer changing. Mathematically, $\mathbf{K}$ and $\mathbf{f}$ at the initial stage, $\mathbf{r}=\mathbf{r}^{0}$, are given as

$$
\begin{aligned}
& \mathbf{K}=\left.\frac{\partial^{2} E_{\mathrm{T}}}{\partial \mathbf{r} \partial \mathbf{r}}\right|_{\mathbf{r}=\mathbf{r}^{0}}=\left.\frac{\partial^{2} \Phi_{\mathrm{T}}}{\partial \mathbf{r} \partial \mathbf{r}}\right|_{\mathbf{r}=\mathbf{r}^{0}}, \\
& \mathbf{f}=-\left.\frac{\partial E_{\mathrm{T}}}{\partial \mathbf{r}}\right|_{\mathbf{r}=\mathbf{r}^{0}}=\mathbf{F}-\left.\frac{\partial \Phi_{\mathrm{T}}}{\partial \mathbf{r}}\right|_{\mathbf{r}=\mathbf{r}^{0}},
\end{aligned}
$$

where $\mathbf{F}$ is the applied external force. Let $\mathbf{r}^{i}$ and $\mathbf{F}^{i}$ denote the position of the $i$ th atom and the external force acting on atom $i$, respectively. For a system composed of $N$ atoms, $\Phi_{\mathrm{T}}$ and $\mathrm{E}_{\mathrm{T}}$ are linked via the following relation

$$
E_{\mathrm{T}}\left(\mathbf{r}^{1}, \mathbf{r}^{2}, \ldots, \mathbf{r}^{N}\right)=\Phi_{T}\left(\mathbf{r}^{1}, \mathbf{r}^{2}, \ldots, \mathbf{r}^{N}\right)-\sum_{i=1}^{N} \mathbf{F}^{i} \cdot \mathbf{r}^{i} .
$$

The potential energy is calculated using the manybody Rafii-Tabar and Sutton (RS) [13] interatomic potentials, a proper potential function for binary systems. RS potentials have been obtained by the modification of Sutton-Chen (SC) potentials [14], which are valid for monoatomic metals only. The SC potential is an empirical many-body, long-range potential of a Finnis and Sinclair [15] type, which combines the short-range interaction feature of Finnis-Sinclair with a Van der Waals tail, suitable for accounting for the long-range interactions. The many-body Finnis and Sinclair potentials describe the cohesion in metals and, in contrast to pair potential functions, are capable of modeling the relaxation at the free surfaces of metals. These capabilities are due to the addition of a cohesive many-body functional incorporating kinetic energy including lattice vibrations and Coulomb and exchange energies besides pairwise contributions. SC potential has proved useful in some previous studies pertinent to nanovoids, cracks, and inclusion within thin films [16-18]. For demonstration, the values of RS potential function parameters for $\mathrm{Cu}$ are given in Table 1 , where $\varepsilon$ is a parameter with the dimension of energy, $n$ and $m$ are positive integers, and $c$ is a positive dimensionless parameter scaling the attractive terms.

Associated with two interacting atoms, $i$ and $j, \mathbf{f}^{i}, \mathbf{f}$, and $\mathbf{K}^{i j}$, where $i, j=1,2, \ldots, N$, are calculated and substituted into Eq. (3) from which the atomic positions are obtained. The details of the formulations for the stiffness matrix and the force vector components are given in Appendix A.
Table 1 Parameters associated with the RS potential function for $\mathrm{Cu}$ fcc metal.

\begin{tabular}{lrrrrr}
\hline Element & $\boldsymbol{a}(\AA)$ & $\boldsymbol{\varepsilon}(\mathrm{eV})$ & $\boldsymbol{c}$ & $\boldsymbol{m}$ & $\boldsymbol{n}$ \\
\hline $\mathrm{Cu}$ & 3.61 & $1.2386 \mathrm{E}-02$ & 39.755 & 6 & 9 \\
\hline
\end{tabular}

\section{Results and discussion}

A three-dimensional simulation technique based on lattice statics in conjunction with RS interatomic potential function for the treatment of an ultrathin fcc film has been developed. Note that the description of the model, including the dimensions and orientation of the film, has been given in Section 2.1. The validity of the applied methodology is substantiated using the first principles DFT calculation in Section 3.1. In Section 3.2, the barrier height energies (in electron volt), $\Delta Q$, along possible outof-plane paths are calculated. Throughout this work, the interstitial atom is placed along the $x_{3}$-axis on any of the (101) planes that are parallel to the free surface of the film. For demonstration, the results are presented for $\mathrm{Cu}$ bulk in Section 3.1 and $\mathrm{Cu}$ film in Section 3.2.

\subsection{First principles DFT calculation of the barrier height energy for the interstitial mechanism of diffusion in Cu bulk}

In this section, the interstitial mechanism of self-interstitial diffusion in $\mathrm{Cu}$ bulk is addressed via ab initio based on DFT. For the verification of the validity of the proposed approach, the results of the ab initio calculations are then compared with those computed by the present method. In this study, the interstitial sites are considered to be of octahedral type as shown in Figure 1. If a diffusing OSIA is to jump to its nearest neighbor, it must pass through point $S$ between atoms labeled 1 and 2, as demonstrated in Figure 1. Thus, the barrier height energy associated with self-interstitial diffusion is closely related to a local dilatation of the lattice. It should be noted that in fcc bulks, the above-mentioned point $S$ is the saddle point $[1,2]$; this point is midway between atoms 1 and 2 .

To calculate the barrier height energy via the proposed lattice statics approach, a model consisting of 3960 atoms is considered. The periodic boundary condition is applied in directions [101], [010], and [101]. At the first step, the interstitial atom is inserted at the interstitial site and the simulation cell is allowed to relax. Subsequently, $E^{I}$ is computed from Eq. (2). In the next step, 
the initial position of the interstitial atom is changed to the saddle point, and after relaxation, $E^{S}$ is determined using Eq. (2). Finally, the activation energy of the system at the saddle point is obtained from Eq. (1); it turns out to be $\Delta Q=0.11 \mathrm{eV}$.

For the verification purposes, the DFT-based Vienna $a b$ initio simulation program [19-22] is used. To model $\mathrm{Cu}$ bulk in the presence of an interestitial atom, a supercell containing 65 atoms with the periodic boundary condition of the simple cubic type is considered. DFT calculations are performed using the projector augmented wave potentials $[23,24]$ with the exchange and correlation effects of the generalized gradient approximation. The Brillouin zone (BZ) integration is made using a $4 \times 6 \times 4 k$-mesh centered at $\Gamma$. To ensure an accurate treatment of the $\mathrm{Cu}$ potential, a plane wave basis set with the energy cutoff of $355 \mathrm{eV}$ for the expansion of the electronic orbitals is used. The ionic ground state is generated using a conjugategradient algorithm with the convergence of $10^{-4} \mathrm{eV}$. The diffusion of the interstitial atom from the octahedral site to the saddle point (point $S$ in Figure 1) goes through four steps. After each step, the system is allowed to relax; recall that the diffusing atom is constrained along the diffusion path. The variation of formation energy due to the migration of diffusing atom is shown in Figure 3. As observed, the activation energy obtained using first principles DFT calculations is $0.18 \mathrm{eV}$, which is in reasonable agreement with the value of $0.11 \mathrm{eV}$ calculated by the proposed lattice statics approach.

\subsection{Surface and interface effects on the interstitial mechanism of diffusion in an ultrathin Cu film}

Throughout this section, an effort is given toward the determination of energetically preferable pathways for

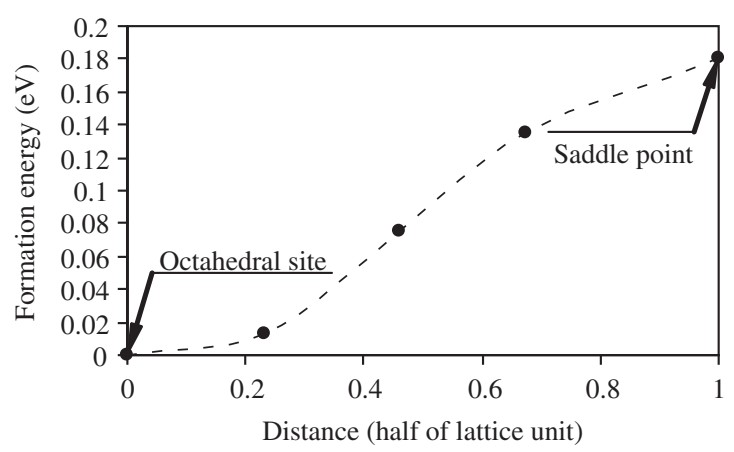

Figure 3 The first principles DFT results of the formation energy of a diffusing self-interstitial atom in Cu bulk. the interstitial mechanism of diffusion in an ultrathin $\mathrm{Cu}$ film bonded to a rigid substrate. The determination of the preferred out-of-plane interstitial diffusion toward the free surface and the rigid substrate based on calculation of the pertinent $\Delta Q$ is of particular interest. As stated in Section 2.1, it is assumed that the orientation of the free surface is (101), and so the out-of-plane path for the interstitial atom is along the [101] direction. In this section, the critical issue is to examine the effects of the film free surface/ film-rigid substrate interface on the barrier height energy. To this end, the interstitial atom is placed at various depths, $x_{3} / a=(-3+0.5 j) \sqrt{2} / 2$, with $j=0,1,2, \ldots, 12$, and the corresponding formation energies are calculated. Note that the free surface and the film-rigid substrate interface are located at $x_{3} / a= \pm 3 \sqrt{2}$, respectively. It should be recalled that, as verified via DFT and the proposed method in Section 3.1, point $S$ for the bulk is the saddle point. However, for the above-mentioned cases, point $S$ may or may not be the saddle point. This matter is discussed in the remainder of this section.

The calculated variation of the formation energy of the interstitial atom at the above-mentioned depths, which correspond to different octahedral sites and points $S$, is presented in Figure 4. In this figure, the letters $O$ and $S$ refer to the position of the interstitial atom when located at the octahedral site and point $S$, respectively. As can be seen, except for the case where $x_{3} / a=2.5 \sqrt{2} / 2$ (point S6) is a saddle point, all the other points $x_{3} / a=(-3+0.5 j) \sqrt{2} / 2$, with $j=1,3, \ldots, 9(S 1, S 2, \ldots, S 5)$ are the interstitial sites. It is observed that the formation energy for $S 5$ is slightly higher than the nearly equal energies pertinent to the sites $S 1, S 2$, $S 3$, and $S 4$. It is evident that the influence of the rigid substrate on the energies for the interstitial sites $S 1$ through $S 5$ is dominant. For further evidence of the remarkable influence of the rigid substrate, attention should be paid to the comparison of formation energy versus the normalized

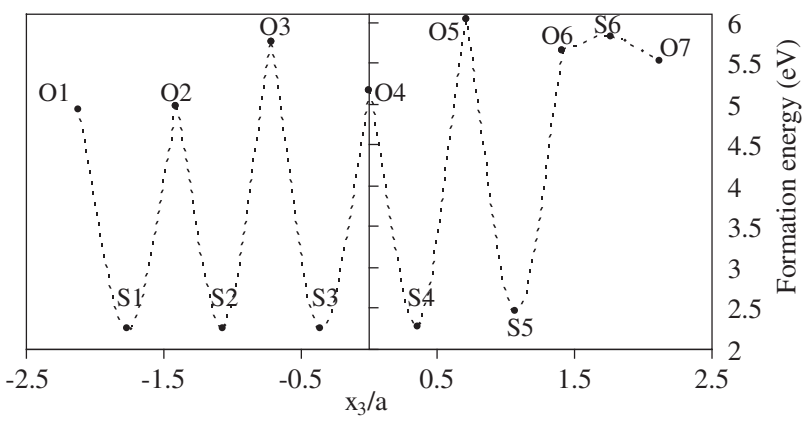

Figure 4 Variation of the formation energy of a diffusing selfinterstitial atom within the ultrathin Cu film consisting of 24 atomic layers. Different depths, $x_{3} / a=(-3+0.5 j) \sqrt{2} / 2$, with $j=0,1,2, \ldots, 12$ for the position of the interstitial atom have been examined. 
distance from the free surface, $X_{3} / a$ for three different film thicknesses consisting of 20, 22, and 24 atomic layers (shown in Figure 5). In all of these cases, the positions of points $S 6$ (in a 24-layer-thick film), $S^{\prime} 6$ (in a 22-layer-thick film), and $S^{\prime \prime} 6$ (in a 20-layer-thick film) have the same distance from the free surface, $X_{3} / a=3.5 \sqrt{2} / 2$. It is clearly seen that the formation energy level for the interstitial atom at the mentioned position from the free surface decreases with decreasing distance from the rigid substrate. Therefore, in the case of 24-layer film, not only the effect of the rigid substrate on the interstitial atom at $S 6$ is small, but it also corresponds to a saddle point as in the bulk. More specifically, the trend of the migration energy of the interstitial atom from $x_{3} / a=\sqrt{2}$ to $x_{3} / a=3 \sqrt{2} / 2$ is qualitatively similar to that of the bulk discussed in the previous section. In other words, the effect of the filmrigid substrate interface that extends to $x_{3} / a=\sqrt{2}$ alters the saddle point and the stable position of the interstitial atom in the film as compared with the corresponding bulk material. It thus appears that the qualitative arguments of diffusion phenomenon can be different from one situation to another. As stated in [11], the geometric considerations stemming from the crystallography of the material and the energetics associated with the atomic-level bonding can result in different qualitative behaviors owing to traversing from the interstitial sites.

The energy required for the migration of the interstitial atom from an initial interstitial site to the next in the direction of the arrow are given within the parenthesis as follows: $S 1 \stackrel{(2.73 \mathrm{eV})}{\rightarrow} S 2 ; S 2 \stackrel{(2.73 \mathrm{eV})}{\rightarrow} S 1 ; S 2 \stackrel{(3.49 \mathrm{eV})}{\rightarrow} S 3 ; S 3 \stackrel{(3.49 \mathrm{ev})}{\rightarrow} S 2$; $S 3 \stackrel{(2.91 \mathrm{ev})}{\rightarrow} S 4 ; S 4 \stackrel{(2.89 \mathrm{ev})}{\rightarrow} S 3 ; S 4 \stackrel{(3.76 \mathrm{ev})}{\rightarrow} S 5 ; S 5 \stackrel{(3.57 \mathrm{ev})}{\rightarrow} S 4$. It was found that the barrier height energy from the interstitial

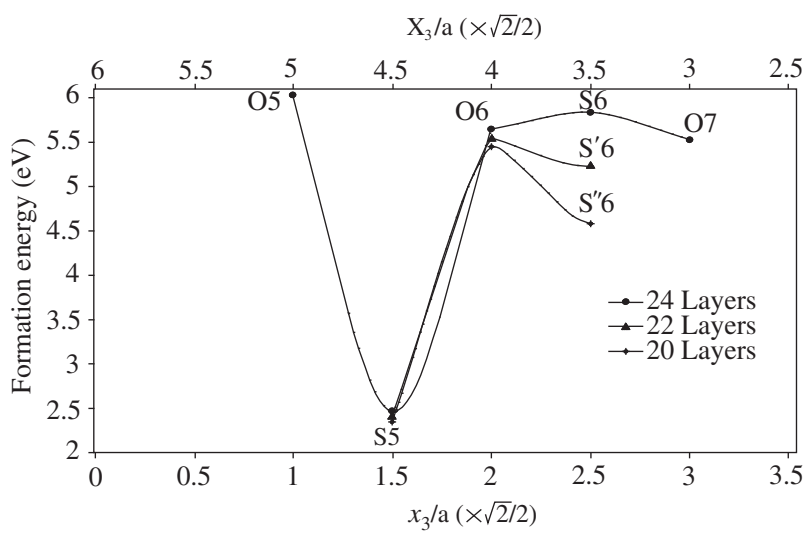

Figure 5 Comparison of the variations of the formation energies versus depth of the interstitial atom for three cases of 20-, 22-, and 24-layer-thick films. $X_{3} / a$ is the normalized distance from the free surface, whereas $x_{3} / a=(-3+0.5 j) \sqrt{2} / 2$ with $j=8,9, \ldots, 12$ measures the normalized distance from the center of the film. site $S 2$ to the interstitial sites $S 1$ and $S 3$ are 2.73 and $3.49 \mathrm{eV}$, respectively. Thus, if the interstitial atom is at point $S 2$, then its migration toward the rigid substrate is more favorable than its migration toward the free surface. The calculated barrier height energies for the paths $S 3 \rightarrow S 2$, $S 3 \rightarrow S 4, S 4 \rightarrow S 3$, and $S 4 \rightarrow S 5$ elucidate that the interstitial atom traps between the two layers located on either sides of the midlayer (between layers $x_{3} / a= \pm \sqrt{2} / 4$ ) because the required activation energy for crossing the midplane of the film is lower than the required energy for moving out of this zone and subsequently migrating toward either the free surface or the rigid substrate.

The examination of the barrier height energies for interstitial atom diffusion pertinent to the paths $S 5 \stackrel{(3.57 \mathrm{ev})}{\rightarrow} S 4$ and $S 5 \stackrel{(3.37 \mathrm{ev})}{\rightarrow} 07$ reveals that the preferred direction of the diffusion is toward the free surface because it requires $0.2 \mathrm{eV}$ lower energy than that required for migrating toward the substrate. When the interstitial atom is located at the octahedral site, $06\left(x_{3}=\sqrt{2} a\right)$, an interesting phenomenon is encountered. In contrast to the previous cases in which the octahedral site was the saddle point, in this case, point $S 6$ coincides with the saddle point. In fact, the trend of diffusion path from 06 to $S 6$ to $O 7$ is similar to that of the $\mathrm{Cu}$ bulk obtained via the present approach as well as the $a b$ initio DFT in the previous section (Figure 3). Recall that the calculated barrier height energy corresponding to the migration from the octahedral site to the saddle point $S$ for the Cu bulk was $0.11 \mathrm{eV}$ by the current method and $0.18 \mathrm{eV}$ via ab initio, whereas the calculated energy of migration from 06 to $S 6$ for $\mathrm{Cu}$ thin film is $0.19 \mathrm{eV}$. It thus seems that the free surface does not change the position of the saddle point, but it increases the needed energy to cross point $S$ as compared with the bulk material.

\section{Concluding remarks}

A new simulation based on the method of lattice statics, which incorporates RS interatomic potential, was used to predict and explain diffusion phenomenon in ultrathin fcc film. The main effort was focused on assessing the effects of the boundary conditions, consisting of the free surface and the rigid substrate, on the self-interstitial diffusion at various depths from the free surface via the interstitial mechanism. It was shown that the distance of the point defect from the free surface plays an essential role in the results. To ensure reasonable precision and numerical convergence, the first and second nearest neighbors had been incorporated in the computations. 
A comparison of the first principles DFT-calculated barrier height energy associated with the interstitial mechanism of diffusion in $\mathrm{Cu}$ bulk with the result obtained by the proposed method verified the capability of the current approach. In the present study, an ultrathin $\mathrm{Cu}$ film, the free surface of which is (101), was simulated. Except for the case where the interstitial atom was located at $x_{3} / a=\sqrt{2}$ and $3 \sqrt{2} / 2$, the octahedral sites were the saddle points. However, when the interstitial atom is at $x_{3} / a=\sqrt{2}$ and diffuses toward the free surface, the saddle point occurs at point $S$, as for the $\mathrm{Cu}$ bulk. The obtained results explain quantitatively that if the interstitial atom is placed at $x_{3} / a=-1.5 \sqrt{2} / 2$, the diffusion toward the filmrigid substrate interface is preferred. The study of diffusion next to the midplane of the 24-layer-thick film shows that the interstitial atom gets trapped between the two layers located on either sides of the midplane, but when the interstitial atom is located at $x_{3} / a=1.5 \sqrt{2} / 2$, a migration toward the free surface takes place.

Acknowledgements: This work was in part supported by the Sharif University of Technology.

\section{Appendix}

For a specimen consisting of $N$ interacting binary atoms, in the context of the RS interatomic potential function, the $\alpha \beta$ th component of the stiffness associated with the $i$ th and $j$ th atoms is calculated as

$$
\begin{aligned}
& \left.k_{\alpha \beta}^{i j}\right|_{i \neq j}=\frac{1}{2} \frac{\mathrm{r}_{\alpha}^{i j} \mathrm{r}_{\beta}^{i j}}{\left(\mathrm{r}^{i j}\right)^{4}}\left\{-\hat{p}_{i} \hat{p}_{j} n^{A A} \varepsilon^{A A} \vartheta(A, n)\left(1+n^{A A}\right)\right. \\
& +\hat{p}_{i} \hat{p}_{j} n^{A A} \varepsilon^{A A} \vartheta(A, n)\left(\delta_{\alpha \beta} \frac{\left(r^{i j}\right)^{2}}{r_{\alpha}^{i j} r_{\beta}^{i j}}-1\right)-\hat{q}_{i} \hat{q}_{j} n^{B B} \varepsilon^{B B} \vartheta(B, n)\left(1+n^{B B}\right) \\
& +\hat{q}_{i} \hat{q}_{j} n^{B B} \varepsilon^{B B} \vartheta(B, n)\left(\delta_{\alpha \beta} \frac{\left(r^{i j}\right)^{2}}{r_{\alpha}^{i j} r_{\beta}^{i j}}-1\right)+\left(\hat{p}_{i} \hat{q}_{j}+\hat{p}_{j} \hat{q}_{i}\right) \\
& {\left[\left(-\frac{1}{4}\left(n^{A A}+n^{B B}\right)^{2}+\frac{1}{2}\left(n^{A A}+n^{B B}\right)\left(\delta_{\alpha \beta} \frac{\left(r^{i j}\right)^{2}}{r_{\alpha}^{i j} r_{\beta}^{i j}}-2\right)\right)\right.} \\
& \left.\sqrt{\varepsilon^{A A} \varepsilon^{B B} \vartheta(A, n) \vartheta(B, n)}\right] \\
& -d^{A A} \hat{p}_{i} \frac{1}{\sqrt{\sum_{j \neq i}\left(\hat{p}_{j} \vartheta(A, m)+\hat{q}_{j} \sqrt{\vartheta(A, m) \vartheta(B, m)}\right)}} \\
& \left(-\hat{p}_{j} m^{A A} \vartheta(A, m)\left(1+m^{A A}\right)+\hat{p}_{j} m^{A A} \vartheta(A, m)\left(\delta_{\alpha \beta} \frac{\left(r^{i j}\right)^{2}}{r_{\alpha}^{i j} r_{\beta}^{i j}}-1\right)-\hat{q}_{j}\right. \\
& {\left[\left(\frac{1}{4}\left(m^{A A}+m^{B B}\right)^{2}-\frac{1}{2}\left(m^{A A}+m^{B B}\right)\left(\delta_{\alpha \beta} \frac{\left(\mathrm{r}^{i j}\right)^{2}}{\mathrm{r}_{\alpha}^{i j} \mathrm{r}_{\beta}^{i j}}-2\right)\right)\right.} \\
& \sqrt{\vartheta(A, m) \vartheta(B, m)}])
\end{aligned}
$$$$
\begin{aligned}
& -d^{B B} \hat{q}_{i} \frac{1}{\sqrt{\sum_{j \neq i}\left(\hat{q}_{j} \vartheta(B, m)+\hat{p}_{j} \sqrt{\vartheta(A, m) \vartheta(B, m)}\right)}} \\
& \left(-\hat{q}_{j} m^{B B} \vartheta(B, m)\left(1+m^{B B}\right)+\hat{q}_{j} m^{B B} \vartheta(B, m)\left(\delta_{\alpha \beta} \frac{\left(r^{i j}\right)^{2}}{r_{\alpha}^{i j} r_{\beta}^{i j}}-1\right)+\hat{p}_{j}\right. \\
& {\left[\left(-\frac{1}{4}\left(m^{A A}+m^{B B}\right)^{2}+\frac{1}{2}\left(m^{A A}+m^{B B}\right)\left(\delta_{\alpha \beta} \frac{\left(r^{i j}\right)^{2}}{r_{\alpha}^{i j} r_{\beta}^{i j}}-2\right)\right)\right.} \\
& \sqrt{\vartheta(A, m) \vartheta(B, m)}])\}, \\
& k_{\alpha \beta}^{i i}=-\sum_{j \neq i} k_{\alpha \beta}^{i j},
\end{aligned}
$$

where $\alpha, \beta \equiv \chi, y$, or $z ; i, j=1,2, \ldots, N$; and $r_{\alpha}^{i j}$ is the $\alpha$ th component of $\mathbf{r}^{i j}=\mathbf{r}^{j}-\mathbf{r}^{i}$. In Eq. (A1), $\vartheta(A, n)=\frac{\left(a^{A A}\right)^{n^{A A}}}{\left(r^{i j}\right)^{n^{A A}}}, d^{A A}=\varepsilon^{A A} c^{A A}$, and $\vartheta(A, m), \vartheta(B, n), \vartheta(B, m)$, and $d^{B B}$ can be obtained in a similar manner. $A$ and $B$ refer to types $A$ and $B$ atoms, respectively. $\delta_{\alpha \beta}$ is the Kronecker delta. The site occupancy operator, $\hat{p}_{i}$, is equal to 1 when site $i$ is occupied by an $A$ atom, and it is 0 when site $i$ is occupied by a $B$ atom. $\hat{q}_{i}$ is determined as $\hat{q}_{i}=1-\hat{p}_{i}$. Moreover, the $a$ th component of the force on the $i$ th atom is obtained from

$$
\begin{aligned}
\mathrm{f}_{\alpha}^{i}= & -\frac{1}{2} \sum_{j \neq i} \frac{\mathrm{r}_{\alpha}^{i j}}{\left(\mathrm{r}^{i j}\right)^{2}}\left\{\hat{p}_{i} \hat{p}_{j} n^{A A} \varepsilon^{A A} \vartheta(A, n)+\hat{q}_{i} \hat{q}_{j} n^{B B} \varepsilon^{B B} \vartheta(B, n)\right. \\
& +\frac{1}{2}\left(\hat{p}_{i} \hat{q}_{j}+\hat{p}_{j} \hat{q}_{i}\right)\left(n^{A A}+n^{B B}\right) \sqrt{\varepsilon^{A A} \varepsilon^{B B} \vartheta(A, n) \vartheta(B, n)}
\end{aligned}
$$




$$
\begin{aligned}
& -d^{A A} \hat{p}_{i} \frac{1}{\sqrt{\sum_{j \neq i}\left(\hat{p}_{j} \vartheta(A, m)+\hat{q}_{j} \sqrt{\vartheta(A, m) \vartheta(B, m)}\right)}} \\
& {\left[\hat{p}_{j} m^{A A} \vartheta(A, m)+\frac{1}{2} \hat{q}_{j}\left(m^{A A}+m^{B B}\right) \sqrt{\vartheta(A, m) \vartheta(B, m)}\right]}
\end{aligned}
$$

$$
\begin{aligned}
& -d^{B B} \hat{q}_{i} \frac{1}{\sqrt{\sum_{j \neq i}\left(\hat{q}_{j} \vartheta(B, m)+\hat{p}_{j} \sqrt{\vartheta(A, m) \vartheta(B, m)}\right)}} \\
& \left.\left[\hat{q}_{j} m^{B B} \vartheta(B, m)+\frac{1}{2} \hat{p}_{j}\left(m^{A A}+m^{B B}\right) \sqrt{\vartheta(A, m) \vartheta(B, m)}\right]\right\} .
\end{aligned}
$$

\section{References}

[1] Shewmon PG. Diffusion in Solids. Bever MB, Michaels AS, Shank ME, Wert CA, Mehl RF, Eds. McGraw-Hill: New York, 1963, pp. 41-60.

[2] Girifalco LA, Welch DO. Point Defects and Diffusion in Strained Metals. Gordon and Breach Science Publishers: New York, 1967.

[3] Halicioglu T, Barnett DM. Surf. Sci. 1999, 441, 265-269.

[4] Xia J, Hu W, Yang J, Ao B, Wang X. Phys. Status Solidi B 2006 243, 579-583.

[5] Ortega MG, Ramos de Debiaggi SB, Monti AM. Phys. Status Solidi B 2002, 234, 506-521.

[6] Haftbaradaran H, Song J, Curtin WA, Gao H. J. Power Sources 2010, 196, 361-370.

[7] Angelo JE, Moody NR, Baskes MI. Model. Simul. Mater. Sci. Eng. 1995, 3, 289-307.

[8] LaBrosse MR, Chen L, Johnson JK. Model. Simul. Mater. Sci. Eng. 2010, 18, 015008.

[9] Young Jr. FW. J. Nucl. Mater. 1978, 69/70, 310-330.

[10] Nordlund K, Keinonen J, Ghaly M, Averback RS. Nature (London) 1999, 398, 49-51.
[11] Phillips R. Crystals, Defects, and Microstructures: Modeling Across Scales. Cambridge University Press: Cambridge, 2001.

[12] Nomura M, Vitek V. Interface Sci. 2003, 11, 99-109.

[13] Rafii-Tabar H, Sutton AP. Philos. Mag. Lett. 1991, 63, 217-224.

[14] Sutton AP, Chen J. Philos. Mag. Lett. 1990, 61, 139-146.

[15] Finnis MW, Sinclair JE. Philos. Mag. A 1984, 50, 45-55.

[16] Shodja HM, Tehranchi A. Philos. Mag. 2010, 90, 1893-1913.

[17] Shodja HM, Kamalzare M. Comput. Mater. Sci. 2009, 45, 275-284.

[18] Shodja HM, Pahlevani L, Hamed E. Mech. Mater. 2007, 39, 803-818.

[19] Kresse G, Hafner J. Phys. Rev. B 1993, 47, 558-561.

[20] Kresse G, Hafner J. Phys. Rev. B 1994, 49, 14251-14269.

[21] Kresse G, Furthmüller J. Comput. Mater. Sci. 1996, 6, 15-50.

[22] Kresse G, Furthmüller J. Phys. Rev. B 1996, 54, 11169-11186.

[23] Kresse G, Joubert J. Phys. Rev. B 1999, 59, 1758-1775.

[24] Blöchl PE, Phys. Rev. B 1994, 50, 17953-17979. 